\title{
Salt Induced Modulation on Physio-Biochemical Attributes in Okra (Abelmoschus esculentus (L.) Moench
}

\author{
Rituparna Munshi ${ }^{1}$, Ajanta Debbarma ${ }^{1}$, Pinaki Acharyya ${ }^{1 *}$, Arup Chattopadhyay ${ }^{3}$, \\ Pritam Mishra ${ }^{1}$, Lopamudra Chakraborty ${ }^{1}$ and Biswanath Sahoo ${ }^{2}$
}

\author{
${ }^{1}$ Department of Horticulture, Institute of Agricultural Science, University of Calcutta,Kolkata- \\ 700019, West Bengal, India \\ ${ }^{2}$ Krishi Vigyan Kendra (OUAT), Ranital, Bhadrak-756111, Odisha, India \\ ${ }^{3}$ Department of Vegetable Science, Faculty of Horticulture, Bidhan Chandra Krishi \\ Viswavidyalaya, Mohanpur-741246, West Bengal, India \\ *Corresponding author
}

\section{Keywords \\ Okra, Salinity \\ tolerance, \\ Germination, \\ Growth, Proline, \\ Protein, Isozyme \\ activity \\ Article Info \\ Accepted: \\ 20 May 2019 \\ Available Online: \\ 10 June 2019}

\section{A B S T R A C T}

A field investigation was carried out in the shade net house at the Agricultural Experimental Farm of Calcutta University at Baruipur, 24-Parganas (South) during MarchJune 2017 and germination tests as well as enzymatic assay were carried out in the Department of Horticulture, Institute of Agriculture Science, University of Calcutta. Out of the 19 accessions of okra under study, five genotypes were selected after screening which could sustain the salinity levels at $50 \mathrm{mM}, 100 \mathrm{mM}$ and $150 \mathrm{mM}$ viz. Arka Anamika, Deepika, Shivani hybrid, Panchvati and Japani Jhar. A two way factorial layout was laid in CRD utilizing polybags for better realization of results and having two replicates for each of the treatment combination. The experiment comprised of 20 treatments. The germination percentage as well as the shoot length was observed to be highest in the variety Deepika under $150 \mathrm{mM}$. The root length was high in Japani Jhar at 100 and $150 \mathrm{mM}$. The number of primary branches per plant was high in Shivani Hybrid under 150 $\mathrm{mM}$. The plant height was high in Deepika under all salinity levels while the inter-nodal length was found to be less in Panchvati under control, 50mM and 100mM. Arka Anamika also exhibited more number of leaves than rest of the genotypes at all levels of salinity. Under 50mM, and $100 \mathrm{mM}$ Shivani Hybrid was the earliest to flower while under $150 \mathrm{mM}$ it was Japani Jhar Similarly, under 50mM and 100mM Japani Jhar showed early 50\% flowering followed by Panchvati and Arka Anamika. The total fresh yield per plant was highest in Japani Jhar at all the levels of salinity followed by Arka Anamika. There was a steady rise in proline and protein content among all the five genotypes with increase in stress. Catalase activity for all the cultivars was higher at $150 \mathrm{mM}$ strength of salt solution and it was noted highest in Arka Anamika closely followed by Panchvati. Peroxidase activity of all the five genotypes could be utilized in future breeding programme to tailor salinity tolerant okra genotypes which could effectively be utilized in marginalized lands. 


\section{Introduction}

High levels of soluble salts in soils or water cause reduction in growth as well as yield of most crops. Salinity can affect several physiological and metabolic processes including protein synthesis, photo- synthetic capacity, photosynthetic efficiency, vacuolar H+-ATPase, respiration, nitrogen metabolism, osmotic potential, enzymatic activities, ionic balance as well as phytohormone regulation. Plant responses to salt stress are complex, which depend on a number of interconnected aspects based on some key physiobiochemical and molecular phenomena. Therefore, elucidation of the mechanisms by which plants perceive and transduce salt stress is quite intricate.

The nutritional imbalance taking place as a result of high amount of sodium $(\mathrm{Na}+)$ may lead to metabolic disorder such as reduction in protein synthesis and enzyme activities. Studies on physiological mechanisms of salt tolerance revealed that plants may reduce damaging effects of salts by controlling salt uptake (Munns and Tester, 2008), reducing damage under excessive ion uptake (Flowers and Yeo, 1995) as well as by osmotic adjustment (Patade et al. 2008; Singh et al. 2010).

Little information is available in the literature on how far different physiological and biochemical attributes in okra are regulated by salt stress. However till date hardly any work has been commenced to screen okra cultivars growing in West Bengal against salinity tolerance.

The present study was conducted to screen the okra cultivars grown in West Bengal under different salinity regimes and evaluate them based on morpho- physiological parameters, growth patterns, fruits characters and yield.

\section{Materials and Methods}

Nineteen (19) diverse genotypes of Okra collected from different sources were employed for the present investigation. All the accessions of okra genotypes were subjected to germination test and parameters like germination percentage, days to $50 \%$ germination, mean germination time, shoot length and root length were recorded from time to time to screen the salinity tolerant genotypes. The okra seeds of designated accessions were germinated on moist blotters on glass plates which were kept in polythene packets containing 20-30 ml of designated salt solution and provided with two rows of holes on the back side. The plates were arranged at $66^{\circ}$ angle on plastic dish-stands which were placed in germinators at 280- 300 for requisite number of days. Day to day observation of the progress in germination was made without disturbing the germinating seeds. The salt solutions of different doses were added at regular intervals for proper result. Hoagland solution was added only in control. 10 seeds of each genotype were used for germination test. The progress was recorded for germination parameters viz. germination percentage, days to $50 \%$ germination, mean germination time, shoot length and root length.

Five tolerant genotypes possessing salinity tolerance as evidenced from survivability count status under varied salinity levels were screened out of the 19 accessions under study and sown in polythene bags subjected to salt solution of $\mathrm{NaCl}$ with Hoagland solution and kept under a shade net house at the Agricultural Experimental Farm of Calcutta University at Baruipur, 24-parganas (South) [880 28'east longitude, 220 22'north latitude] during March-June 2017. Different levels viz. control, $50 \mathrm{mM}, 100 \mathrm{mM}, 150 \mathrm{mM}$ of $\mathrm{NaCl}$ were applied to each unit according to the saturation percentage of soil The salt solution 
was applied in the soil of polythene bag with a $50 \mathrm{mM}$ basal dose and then gradually increased on a regular basis up to $150 \mathrm{mM}$. Hoagland solution was applied as control. A two way factorial layout was laid in CRD having two replicates for each of the treatment combinations. Observations pertaining to plant height, inter-nodal length ,number of leaves, number of primary branches, days to $50 \%$ flowering, days to first flowering, fruit length $(\mathrm{cm})$, fruit diameter (cm),fruit weight ( $\mathrm{g})$,number of seeds per fruit and number of fruits per plant were recorded. Plot Means were used for statistical analysis using the statistical package "Two factor Factorial CRD software" available online (www.ccari.res.in/wasp).

\section{Results and Discussion}

In the beginning 19 accessions of okra collected from different reliable sources were subjected to germination test and related parameters to screen for salinity, the results of which are depicted in Table 1. Observations revealed that five genotypes viz. Japani Jhar, Shivani Hybrid, Deepika, Arka Anamika and Panchvati showed consistently good results for the basic parameters i.e. germination percentage, shoot length and root length and were selected for further screening exercise. In the follow up investigation, the mean germination percentage of all the five genotypes gradually decreased with increased salinity(Table 2). Under $50 \mathrm{mM}$ and $100 \mathrm{mM}$ solution higher germination percentage was observed in Japani Jhar(156.49, 124.27) while under $150 \mathrm{mM}$ higher success rate was observed in Shivani Hybrid(120.488). Our findings are in accordance with Zapata et al.,(2004). Lower levels of salinity delayed germination, whereas higher levels reduced the final percentage of seed germination, which could be attributed to osmotic stress or specific ion toxicity (Huang and Redmann, 1995). The days to germination gradually increased with increase in salinity levels, Deepika performed better over others at 50 and $100 \mathrm{mM}(10.6,12.3)$ while under $150 \mathrm{mM}$ it was Shivani Hybrid (14.3). Younis et al., (1991) reported that low moisture content under salt stress condition cause cessation of metabolism or inhibition of certain steps in metabolic sequences of germination. Moreover, several reports suggest hypersaline environment caused delayed germination (Prado et al., 1995) by reducing hydrolytic enzyme activities and retarding the mobilization rate of metabolites. Saline stress limits hydrolysis of food reserves from storage tissue and impairs their translocation from storage tissue to developing embryo axis (Dubey, 2005 and De Lacerda et al., 2003). The mean germination time gradually increased with rise in salinity level for all the accessions under study and was lowest in Arka Anamika under control (6.3) and at all other salinity levels over rest of the cultivars. The mechanism of inhibition of germination or slowing down of seedling growth by $\mathrm{NaCl}$ may be related to radicle emergence due to insufficient water absorbed or may be subscribed to toxic effect on the embryo. The shoot length gradually decreased with increase in salinity levels, was highest in Japani Jhar under control (5.22) as well as under 50 and $100 \mathrm{mM}(4.2,4.02)$. Excessive entry of toxic ions into the plant inhibits radicle and plumule growth by disintegrating their tissues. Growth parameters like seedling shoot length, root length, fresh weight and dry weight exhibited a strong negative correlation with salinity. Further, the growth attributes may be linked with the function of PGRs, stimulating the growth and development. The water potential also gets reduced in the root zone with the increasing salinity level which lowers the cell turgidity and this in turn hampers the cell differentiation and cell elongation which leads to inhibition of growth in cultivars grown under saline condition. Similarly, decreases in root length in variable 
proportions were observed for all the cultivars with increased salinity levels. Japani Jhar was having higher root length over others at 100 and $150 \mathrm{mM}$ (38.84, 30.48). Similar reports were also communicated by Abbas et al.,(2013) suggesting that germinated seedlings under varied salinity level followed attenuating pattern in terms of seedling root and shoot length, fresh and dry weight.

All the five tolerant genotypes were grown in polybags treated with variable salt solution of $\mathrm{NaCl}$ in Hoagland and morphometric characters were recorded as depicted in Table 3. Plant growth is an important character in determining the salt tolerance ability of the plants. The plant height was high in Deepika under control (78.54) as well as under $50 \mathrm{mM}$ (66.76), 100mM (50.58) and 150mM (47.80) respectively. Rest of the varieties too showed a decrease in plant height with respect to increased salinity levels. Though plant height is genetically controlled; environmental factors also have strong influence in the expression of genes as it is very clear from the present investigation that all treatments of salt stress significantly influenced the plant. With increase in salinity, there was failure in activation of the dehydration avoidance mechanism like making root membranes impermeable for toxic ions of $\mathrm{Na}+$ and $\mathrm{Cl}-$. The number of primary branches per plant was high in Arka Anamika (7.88) under control as well as under $50 \mathrm{mM}(7.74)$ and $100 \mathrm{mM}$ (6.22) while it was Shivani Hybrid under $150 \mathrm{mM}$ (6.20). The inter-nodal length was found to be less in Panchvati under control (15.90), 50mM (13.04) and $100 \mathrm{mM}$ (10.52). However, the least internodal length was noted in Japani Jhar (8.54) under 150mM .Number of leaves per plant was higher in Arka Anamika (40.10) and Deepika (40.54) under control. Arka Anamika also exhibited more no. of leaves than rest of the genotypes in rest of the salinity levels. Least no of leaves were observed in Japani Jharunder all the treatments. Days to first flowering was earliest in Panchavati (99.10) which was followed by Deepika (102.50) and Shivani Hybrid (102.24) under control. Under $50 \mathrm{mM}$ and $100 \mathrm{mM}$, Shivani Hybrid was the earliest to flower $(102.00,111.12)$ while under $150 \mathrm{mM}$ it was Japani Jhar (128.28). With increase in salinity level, days to first flowering increased for all the cultivars. Days to $50 \%$ flowering were early in Panchvati under control (108.48) and it was Shivani Hybrid at $50 \mathrm{mM}$ (117.84) and $100 \mathrm{mM}$ (118.14). But under 150mM Japani Jhar was early to flower (140.34). The total fresh yield per plant was highest in Japani Jhar under control $(780.30 \mathrm{~g})$ and at all the levels of salinity ending up at $465.44 \mathrm{~g}$ under $150 \mathrm{mM}$. It was closely followed by Arka Anamika. Results suggest that there was a sharp decline in yield of all the five cultivars under study with increased salinity levels. Similar reports were furnished by workers like Abbas et al., (2013) and others while working on okra who were of the view that severe reduction in morphological attributes including shoot length, fresh plant weight and leaf area takes place under saline situations.

Proline content was recorded highest in Panchvati followed by Deepika under control., 50 and $100 \mathrm{mM}$ salt concentration (Table 4), however under 150mM Deepika was having higher level of proline than Panchvati. Accumulation of proline in plant under saline regime is generally considered as an important phenomenon involved in stress as proline plays a vital role in osmotic adjustment exposed to stress condition as reported in earlier experimental observations. Protein content gradually increased in all the cultivars with increased stress (Table 4). The more tolerant genotypes showed higher protein content and it was highest in Shivani hybrid under $150 \mathrm{mM}(0.454)$ followed by Japani Jhar (0.412). 
Table.1 Screening of 19 accessions of okra towards salinity tolerance based on germination parameters

\begin{tabular}{|c|c|c|c|c|c|}
\hline Genotypes & Parameters & Control & $50 \mathrm{mM}$ & $100 \mathrm{mM}$ & $150 \mathrm{mM}$ \\
\hline \multirow[t]{3}{*}{ Japani jhar } & G.P & 100 & 100 & 100 & 100 \\
\hline & S.L. & 2.18 & 1.15 & 0.9 & 0.7 \\
\hline & R.L. & 24.6 & 14.2 & 10.16 & 6.6 \\
\hline \multirow[t]{3}{*}{ Avantika } & G.P & 80 & 80 & 80 & 100 \\
\hline & S.L. & 1.6 & 0.8 & 0.76 & 0.5 \\
\hline & R.L. & 16.6 & 11.7 & 3.6 & 1.87 \\
\hline \multirow[t]{3}{*}{ Tata } & G.P & 100 & 100 & 80 & 80 \\
\hline & R.L. & 1.97 & 1.06 & 1.04 & 0.93 \\
\hline & S.L. & 21.62 & 9.78 & 6.63 & 5.96 \\
\hline \multirow[t]{3}{*}{ Arka Anamika } & G.P & 100 & 100 & 100 & 100 \\
\hline & S.L. & 2.18 & 1.48 & 1.13 & 0.8 \\
\hline & R.L. & 24.6 & 13.9 & 6.67 & 6.5 \\
\hline \multirow[t]{3}{*}{ Deepika } & G.P & 100 & 100 & 100 & 100 \\
\hline & S.L. & 1.97 & 1.78 & 1.24 & 5.87 \\
\hline & R.L. & 21.62 & 18.33 & 12.88 & 1.13 \\
\hline \multirow[t]{3}{*}{ Sonakshi } & G.P & 100 & 100 & 100 & 100 \\
\hline & S.L. & 1.62 & 1.08 & 0.96 & 1.56 \\
\hline & R.L. & 19.4 & 8.6 & 8.04 & 9 \\
\hline \multirow[t]{3}{*}{ Shiba } & G.P & 100 & 80 & 80 & 100 \\
\hline & S.L. & 2.04 & 1.62 & 0.9 & 1.18 \\
\hline & R.L. & 22.8 & 15.9 & 5.38 & 6.64 \\
\hline \multirow[t]{3}{*}{ Shahiba } & G.P & 100 & 100 & 80 & 80 \\
\hline & S.L. & 2.27 & 1.14 & 0.95 & 0.78 \\
\hline & R.L. & 24.0 & 9.9 & 6.3 & 4.75 \\
\hline \multirow[t]{3}{*}{ Bhindi No 10} & G.P & 20 & 20 & 20 & 20 \\
\hline & S.L. & 2.0 & 0.5 & 0.9 & 0.8 \\
\hline & R.L. & 20.0 & 1.3 & 1.2 & 1.1 \\
\hline \multirow[t]{3}{*}{ No 55} & G.P & 100 & 100 & 80 & 80 \\
\hline & S.L. & 2.52 & 1.28 & 1.12 & 1.12 \\
\hline & R.L. & 23.4 & 8.25 & 5.4 & 5.4 \\
\hline \multirow[t]{3}{*}{ JOKH 6166} & G.P & 100 & 100 & 60 & 60 \\
\hline & S.L. & 1.8 & 0.76 & 1.37 & 1.37 \\
\hline & R.L. & 17.4 & 6.5 & 6.67 & 6.67 \\
\hline \multirow[t]{3}{*}{ NKOH 1001} & G.P & 100 & 80 & 80 & 80 \\
\hline & S.L. & 1.44 & 1.35 & 0.95 & 0.95 \\
\hline & R.L. & 15.9 & 8.25 & 4.75 & 4.75 \\
\hline \multirow[t]{3}{*}{ Utkal Gaurav } & G.P & 100 & 100 & 80 & 80 \\
\hline & S.L. & 1.66 & 1.06 & 0.9 & 0.9 \\
\hline & R.L. & 22.9 & 11.84 & 4.0 & 4.0 \\
\hline \multirow[t]{3}{*}{ Indame } & G.P & 80 & 60 & 60 & 60 \\
\hline & S.L. & 1.66 & 1.03 & 0.9 & 0.9 \\
\hline & R.L. & 22.9 & 7.17 & 4.9 & 4.0 \\
\hline \multirow[t]{3}{*}{ Panchvati } & G.P & 100 & 100 & 100 & 100 \\
\hline & S.L. & 1.86 & 1.14 & 1.10 & 0.68 \\
\hline & R.L. & 21.7 & 15.6 & 10.8 & 4.06 \\
\hline \multirow[t]{3}{*}{ Bhindi Pankaj } & G.P & 100 & 100 & 100 & 100 \\
\hline & S.L. & 1.7 & 0.88 & 0.94 & 0.94 \\
\hline & R.L. & 20.7 & 7.02 & 6.7 & 6.7 \\
\hline \multirow[t]{3}{*}{ Pankaj Bhindi sp. } & G.P & 100 & 100 & 60 & 60 \\
\hline & S.L. & 1.7 & 1.28 & 1.1 & 1.1 \\
\hline & R.L. & 20.7 & 9.54 & 6.6 & 6.6 \\
\hline Shakthi F1 & G.P & 100 & 100 & 80 & 60 \\
\hline & S.L. & 1.62 & 1.4 & 0.95 & 0.4 \\
\hline & R.L. & 17.0 & 10.78 & 4.75 & 3.5 \\
\hline Shivani Hybrid & G.P & 100 & 100 & 100 & 100 \\
\hline & S.L. & 1.62 & 1.08 & 1.56 & 0.96 \\
\hline & R.L. & 19.4 & 9.0 & 8.6 & 8.04 \\
\hline
\end{tabular}

Note. G.P.-germination percentage, R.L.-root length, S.L. - shoot length CD $(\mathbf{P}=\mathbf{0 . 0 5})$ 


\begin{tabular}{|l|c|c|c|}
\hline Parameters & Variety & Salinity level & $\begin{array}{c}\text { Variety x Salinity } \\
\text { level }\end{array}$ \\
\hline $\begin{array}{l}\text { Germination } \\
\text { Percentage }\end{array}$ & 8 & 4 & 15 \\
\hline Shoot length & 0.14 & 0.07 & 0.28 \\
\hline Root length & 0.9 & 0.5 & 1.8 \\
\hline
\end{tabular}

Table.2 Germination parameters of salinity tolerant okra genotypes under study

\begin{tabular}{|c|c|c|c|c|c|}
\hline Genotypes & Parameters & Control & $50 \mathrm{mM}$ & $100 \mathrm{mM}$ & $150 \mathrm{mM}$ \\
\hline \multirow{5}{*}{$\begin{array}{l}\text { Arka } \\
\text { Anamika }\end{array}$} & G.P & 166.33 & 123.86 & 120.44 & 119.38 \\
\hline & $50 \%$ germ. & 7.9 & 11.1 & 13.7 & 15.1 \\
\hline & M.G.T. & 6.3 & 8.2 & 10.15 & 14.35 \\
\hline & S.L. & 4.74 & 3.1 & 3.24 & 1.85 \\
\hline & R.L. & 48.42 & 19.72 & 14.86 & 13.19 \\
\hline \multirow{5}{*}{ Deepika } & G.P & 167.73 & 124.77 & 100.57 & 74.22 \\
\hline & $50 \%$ germ. & 9.3 & 10.6 & 12.3 & 16.6 \\
\hline & M.G.T. & 8.14 & 9.58 & 13.85 & 17.55 \\
\hline & S.L. & 5.06 & 3.47 & 2.69 & 2.45 \\
\hline & R.L. & 36.84 & 35.05 & 13.13 & 13.96 \\
\hline \multirow{5}{*}{ Shivani hybrid } & G.P & 161.14 & 124.31 & 121.67 & 120.48 \\
\hline & $50 \%$ germ. & 6.45 & 11.4 & 13.4 & 14.3 \\
\hline & M.G.T. & 7.86 & 11.88 & 13.45 & 18.01 \\
\hline & S.L. & 4.13 & 2.44 & 2.54 & 2.12 \\
\hline & R.L. & 45.94 & 35.47 & 26.42 & 15.54 \\
\hline \multirow[t]{5}{*}{ Japani jhar } & G.P & 159.13 & 156.49 & 124.27 & 98.86 \\
\hline & $50 \%$ germ. & 9.5 & 12.45 & 13.35 & 17.1 \\
\hline & M.G.T. & 8.05 & 11.8 & 15.74 & 18.28 \\
\hline & S.L. & 5.22 & 4.2 & 4.02 & 1.93 \\
\hline & R.L. & 47.52 & 42.53 & 38.84 & 30.48 \\
\hline \multirow{5}{*}{ Panchvati } & G.P & 161.09 & 128.6 & 103.15 & 100.55 \\
\hline & $50 \%$ germ. & 7 & 11.1 & 12.85 & 18.3 \\
\hline & M.G.T. & 8.3 & 11.95 & 16.28 & 18.46 \\
\hline & S.L. & 3.59 & 2.65 & 2.23 & 1.93 \\
\hline & R.L. & 35.32 & 30.94 & 24.29 & 21.49 \\
\hline
\end{tabular}

\begin{tabular}{|c|c|c|c|c|}
\hline Parameters & CV & CD5\% (A) & CD5\% (B) & A XB \\
\hline G.P & 7.74 & 5.18 & 4.63 & 10.36 \\
\hline 50\%germ. & 2.66 & 0.17 & 0.15 & 0.34 \\
\hline M.G.T. & 3.30 & 0.21 & 0.19 & 0.43 \\
\hline S.L & 17.31 & 0.28 & 0.25 & 0.57 \\
\hline R.L. & 5.40 & 0.83 & 0.74 & 1.67 \\
\hline
\end{tabular}

FACTOR: A- VARIETY, FACTOR B- SALINITY LEVEL, CD AT 5\% LEVEL

NOTE: G.P.: Germination Percentage 50\% germ.: Days to 50\%germination, M.G.T.: Mean germination time,

S.L.: Shoot Length, R.L.: Root Length 
Table.3 Estimation of selected morphological parameters in the screened genotypes of okra towards salinity tolerance

\begin{tabular}{|c|c|c|c|c|c|}
\hline VARIETY & Parameters & Control & $50 \mathrm{mM}$ & $100 \mathrm{mM}$ & $150 \mathrm{mM}$ \\
\hline \multirow{7}{*}{ Shivani Hybrid } & P.H.(cm) & 60.88 & 55.66 & 46.80 & 39.54 \\
\hline & NO .of .Pr.Br/PI. & 4.12 & 3.90 & 3.84 & 6.20 \\
\hline & I.L. (cm) & 16.94 & 14.74 & 14.12 & 11.00 \\
\hline & No. of L/PI. & 38.24 & 36.44 & 29.74 & 24.20 \\
\hline & D.T. F.Fl. & 102.24 & 102.00 & 111.12 & 137.24 \\
\hline & D.T.50\% Fl. & 116.70 & 117.84 & 118.14 & 148.3 \\
\hline & Fr. Y/PI. & 632.52 & 495.44 & 406.14 & 320.40 \\
\hline \multirow{7}{*}{ JapaniJhar } & P.H.(cm) & 61.68 & 52.14 & 44.84 & 37.70 \\
\hline & NO .of .Pr.Br/PI. & 6.15 & 5.87 & 3.80 & 3.88 \\
\hline & I.L. (cm) & 18.04 & 16.1 & 13.84 & 8.54 \\
\hline & No. of $L / P I$. & 32.50 & 28.58 & 25.24 & 19.58 \\
\hline & D.T. F.Fl. & 110.30 & 116.80 & 122.22 & 128.28 \\
\hline & D.T.50\% Fl. & 110.84 & 126.44 & 130.94 & 140.34 \\
\hline & Fr. Y/PI. & 780.30 & 643.86 & 577.44 & 465.44 \\
\hline \multirow{7}{*}{ ArkaAnamika } & P.H.(cm) & 64.64 & 65.94 & 42.94 & 37.24 \\
\hline & NO .of .Pr.Br/PI. & 7.88 & 7.74 & 6.22 & 6.14 \\
\hline & I.L. (cm) & 23.94 & 20.34 & 16.74 & 13.54 \\
\hline & No. of $L / P I$. & 40.10 & 38.04 & 34.42 & 29.94 \\
\hline & D.T. F.Fl. & 100.00 & 112.84 & 128.24 & 138.84 \\
\hline & D.T.50\% Fl. & 116.80 & 121.54 & 134.24 & 158.54 \\
\hline & Fr. Y/PI. & 731.58 & 615.78 & 539.44 & 435.90 \\
\hline \multirow{7}{*}{ Deepika } & P.H.(cm) & 78.54 & 66.76 & 50.58 & 47.80 \\
\hline & NO .of .Pr.Br/Pl. & 5.94 & 6.08 & 6.10 & 3.96 \\
\hline & I.L. (cm) & 21.70 & 16.85 & 11.92 & 9.84 \\
\hline & No. of L/PI. & 40.54 & 35.64 & 25.84 & 25.56 \\
\hline & D.T. F.Fl. & 102.50 & 120.04 & 137.28 & 148.00 \\
\hline & D.T.50\% Fl. & 109.10 & 128.44 & 139.28 & 160.14 \\
\hline & Fr. Y/PI. & 580.64 & 499.22 & 453.76 & 346.16 \\
\hline \multirow{7}{*}{ Panchvati } & P.H.(cm) & 61.40 & 46.96 & 39.84 & 41.80 \\
\hline & No.of.Pr.Br/Pl. & 6.14 & 5.94 & 6.08 & 4.24 \\
\hline & I.L. (cm) & 15.90 & 13.04 & 10.52 & 9.80 \\
\hline & No. of $L / P I$. & 36.42 & 35.50 & 27.24 & 26.84 \\
\hline & D.T. F.Fl. & 99.10 & 116.50 & 126.84 & 135.74 \\
\hline & D.T.50\% Fl. & 108.48 & 119.86 & 139.00 & 152.80 \\
\hline & Fr. Y/PI. & 625.94 & 484.04 & 399.68 & 296.45 \\
\hline
\end{tabular}

\begin{tabular}{|c|c|c|c|c|}
\hline Parameters & CV & CD5\% (A) & CD5\% (B) & A XB \\
\hline P.H & 3.38 & 1.04 & 0.93 & 2.09 \\
\hline No.ofPr.Br/PI & 3.87 & 0.11 & 0.10 & 0.22 \\
\hline I.L. & 1.83 & 0.14 & 0.12 & 0.28 \\
\hline No.of L/Pl & 6.11 & 1.00 & 0.90 & 2.01 \\
\hline D.T.F.Fl. & 2.04 & 1.28 & 1.14 & 2.56 \\
\hline D.T.50\%Fl. & 1.82 & 1.24 & 1.11 & 2.48 \\
\hline Fr. Y/Pl. & 4.17 & 11.28 & 10.09 & 22.17 \\
\hline
\end{tabular}

NOTE: P.H.: Plant height, No. of Pr.Br/PI: Number of primary branch per plant, I.l.: Internodal length, No. of L/PI.: Number of leaves per plant, D.T.F.Fl.: Days to first flowering, D.T.50\% Fl.: Days to 50\% flowering, Fr. Y/PI. : Fresh yield per plant

FACTOR: A- VARIETY, FACTOR B- SALINITY LEVEL, CD AT 5\% LEVEL 
Table.4 Estimation of biochemical attributes in the screened accessions of okra towards salinity tolerance

\begin{tabular}{|c|c|c|c|c|c|}
\hline Genotypes & Parameters & Control & $50 \mathrm{mM}$ & $100 \mathrm{mM}$ & $150 \mathrm{mM}$ \\
\hline \multirow{4}{*}{$\begin{array}{l}\text { Arka } \\
\text { Anamika }\end{array}$} & $\begin{array}{l}\text { Proline Content }(\mu \mu \\
\text { moles/g fresh wt) }\end{array}$ & 0.2 & 0.21 & 0.258 & 0.282 \\
\hline & Total Protein (mg/g) & 0.272 & 0.284 & 0.288 & 0.290 \\
\hline & $\begin{array}{c}\text { Catalase activity }(\Delta \mathrm{OD} \\
240 / \mathrm{min} / \mu \mathrm{g} \text { protein } / \mu \mathrm{g} \\
\text { tissue })\end{array}$ & 0.004 & 0.006 & 0.014 & 0.016 \\
\hline & $\begin{array}{c}\text { Peroxidase } \\
\text { activity }\left(\Delta \mathrm{OD}{ }_{470} / \mathrm{min} / \mu \mathrm{g}\right. \\
\text { protein } / \mu \mathrm{g} \text { tissue })\end{array}$ & 0.03 & 0.046 & 0.05 & 0.06 \\
\hline \multirow[t]{4}{*}{ Deepika } & $\begin{array}{l}\text { Proline Content ( } \mu \mu \\
\text { moles/g fresh wt) }\end{array}$ & 0.666 & 1.004 & 1.14 & 1.38 \\
\hline & Total Protein $(\mathrm{mg} / \mathrm{g})$ & 0.286 & 0.298 & 0.358 & 0.368 \\
\hline & $\begin{array}{c}\text { Catalase activity }(\Delta \mathrm{OD} \\
240 / \mathrm{min} / \mu \mathrm{g} \text { protein } / \mu \mathrm{g} \\
\text { tissue })\end{array}$ & 0.002 & 0.004 & 0.006 & 0.006 \\
\hline & $\begin{array}{c}\text { Peroxidase } \\
\text { activity }\left(\Delta \mathrm{OD}{ }_{470} / \mathrm{min} / \mu \mathrm{g}\right. \\
\text { protein } / \mu \mathrm{g} \text { tissue })\end{array}$ & 0.002 & 0.004 & 0.008 & 0.01 \\
\hline \multirow[t]{4}{*}{$\begin{array}{l}\text { Shivani } \\
\text { hybrid }\end{array}$} & $\begin{array}{l}\text { Proline Content }(\mu \mu \\
\text { moles/g fresh wt) }\end{array}$ & 0.216 & 0.300 & 0.478 & 0.524 \\
\hline & Total Protein (mg/g) & 0.392 & 0.428 & 0.438 & 0.454 \\
\hline & $\begin{array}{c}\text { Catalase activity }(\Delta \mathrm{OD} \\
240 / \mathrm{min} / \mu \mathrm{g} \text { protein } / \mu \mathrm{g} \\
\text { tissue })\end{array}$ & 0.004 & 0.004 & 0.012 & 0.014 \\
\hline & $\begin{array}{c}\text { Peroxidase } \\
\text { activity }\left(\Delta \mathrm{OD}{ }_{470} / \mathrm{min} / \mu \mathrm{g}\right. \\
\text { protein } / \mu \mathrm{g} \text { tissue })\end{array}$ & 0.02 & 0.036 & 0.044 & 0.056 \\
\hline \multirow[t]{4}{*}{ Japani jhar } & $\begin{array}{l}\text { Proline Content ( } \mu \mu \\
\text { moles/g fresh wt) }\end{array}$ & 0.204 & 0.274 & 0.298 & 0.386 \\
\hline & Total Protein $(\mathrm{mg} / \mathrm{g})$ & 0.268 & 0.392 & 0.406 & 0.412 \\
\hline & $\begin{array}{c}\text { Catalase activity }(\Delta \mathrm{OD} \\
240 / \mathrm{min} / \mu \mathrm{g} \text { protein } / \mu \mathrm{g} \\
\text { tissue })\end{array}$ & 0.002 & 0.006 & 0.008 & 0.008 \\
\hline & $\begin{array}{c}\text { Peroxidase } \\
\text { activity }\left(\Delta \mathrm{OD}{ }_{470} / \mathrm{min} / \mu \mathrm{g}\right. \\
\text { protein } / \mu \mathrm{g} \text { tissue })\end{array}$ & 0.004 & 0.008 & 0.018 & 0.022 \\
\hline \multirow[t]{4}{*}{ Panchvati } & $\begin{array}{l}\text { Proline Content ( } \mu \mu \\
\text { moles/g fresh wt) }\end{array}$ & 0.722 & 1.094 & 1.13 & 1.196 \\
\hline & Total Protein (mg/g) & 0.186 & 0.198 & 0.208 & 0.228 \\
\hline & $\begin{array}{c}\text { Catalase activity }(\Delta \mathrm{OD} \\
240 / \mathrm{min} / \mu \mathrm{g} \text { protein } / \mu \mathrm{g} \\
\text { tissue })\end{array}$ & 0.006 & 0.008 & 0.01 & 0.012 \\
\hline & $\begin{array}{c}\text { Peroxidase } \\
\text { activity }\left(\Delta \mathrm{OD}{ }_{470} / \mathrm{min} / \mu \mathrm{g}\right. \\
\text { protein } / \mu \mathrm{g} \text { tissue })\end{array}$ & 0.004 & 0.014 & 0.022 & 0.028 \\
\hline
\end{tabular}




\begin{tabular}{|c|c|c|c|c|}
\hline Parameters & $\mathrm{CV}$ & CD5\% (A) & CD5\% (B) & A XB \\
\hline $\begin{array}{c}\text { Proline Content }(\mu \mu \text { moles} / g \text { fresh } \\
\text { wt) }\end{array}$ & 2.044 & 0.006 & 0.006 & 0.013 \\
\hline Total Protein $(\mathrm{mg} / \mathrm{g})$ & 1.006 & 0.002 & 0.002 & 0.003 \\
\hline $\begin{array}{c}\text { Catalase activity }\left(\Delta \mathrm{OD}_{240} / \mathrm{min} / \mu \mathrm{g}\right. \\
\text { protein } / \mu \mathrm{g} \text { tissue })\end{array}$ & 8.456 & 0.000 & 0.000 & 0.001 \\
\hline $\begin{array}{c}\text { Peroxidase activity }\left(\Delta O D_{470} / \mathrm{min} / \mu \mathrm{g}\right. \\
\left.\text { protein } / \mu \mathrm{g}_{\text {tissue }}\right)\end{array}$ & 6.116 & 0.001 & 0.001 & 0.002 \\
\hline
\end{tabular}

Our findings do agree with that of Abbas et al., (2013). It is of utmost importance that plants maintain protein homeostasis i.e., the balance between protein biosynthesis and degradation under both optimal and stress environmental conditions as under stress, proteins are often attacked by reactive oxygen species causing structural changes that jeopardize their functions leading to their aberration and finally degraded by plant proteolytic enzymes. Catalase activity (Table 4) for all the cultivars were higher at $150 \mathrm{mM}$ strength of salt solution and it was noted highest in Arka Anamika (0.016) closely followed by Panchvati (0.036). There was a gradual rise in catalase activity with increase in salinity levels. Maximum Peroxidase activity(POD) was recorded in plants treated with high $\mathrm{NaCl}$ concentration @ $150 \mathrm{Mm}$ followed by $100 \mathrm{Mm}$ and $50 \mathrm{Mm}$ (Table 4). The plants of Arka Anamika and Shivani Hybrid exhibited maximum percent rise in POD as compared to Panchvati, Japani Jhar and Deepika. Similar findings have been reported by Hussain et al., (2013) and many other workers who were of the view that a strong correlation exists between the salt tolerant condition and antioxidant activity

The present investigation has conclusively brought out some vital information that salt induced stress on okra cultivars greatly affected germination parameters like germination $\%$, days to $50 \%$ germination, mean germination time, shoot and root length as well as the morphological traits and biochemical attributes. All the five screened genotypes viz. Arka Anamika, Deepika, Shivani hybrid, Panchvati and Japani Jhar could be utilized in future breeding programme for development of salinity tolerant okra genotypes which will help in utilization of marginalized lands to a great extent. However, they should be put to multilocational testing to prove their stability. Molecular characterization of such genotypes will be an added advantage as it will help us to identify the genes responsible for imparting the resistance.

\section{Acknowledgement}

The authors are thankful to the University of Calcutta for extending the laboratory and land facility for carrying out the research program.

\section{References}

Abbas Ghulam., Saqib Muhammad., Akhtar Javaid and Basra, S.M. A., 2013, Salinity accumulation and distribution during shoot and leaf development in two sorghum genotypes under salt stress. Environ. Exp. Bot., 49: 107-120.

De Lacerda, C.F., Cambraia J., Oliva, M.A., Ruiz, H.A. and Prisco, J.T., 2003, Solute tolerance potential of two Acacia species at early seedling stage. Pak. J. Agri. Sci., 50(4): 683-688.

Dubey, R. S., 2005, Photosynthesis in plants under stress full conditions. In: M. Pessarakli, ed. Photosynthesis, CRC Press, New York, NY, pp. 717-718.

Flowers, T. J. and Yeo, A. R., 1995, Breeding 
for salinity resistance in crop plants: where next? Australian Journal of Plant Physiology, 22: 875- 884.

Huang, J. and Reddman, R.E. 1995, Salt tolerance of Hordeum and Brassica species during germination and early seedling growth. Can. J. Plant Sci., 75: 815-819.

Hussain, S., Khaliq, A., Matloob, A., Wahid, M. A. and Afzal, I., 2013, Germination and growth response of three wheat cultivars to $\mathrm{NaCl}$ salinity. Soil and Environment, 32: 36-43.

Munns, R. and Tester, M., 2008, Mechanisms of salinity tolerance. Annu. Rev. Plant Biol., 59: 651-681.

Patade, V. Y., Suprasanna, P. and Bapat, V. 2008, Effects of salt stress in relation to osmotic adjustment on sugarcane (Saccharum officinarum L.) callus cultures. Plant Growth Re- gul, 55: 169-173.

Prado, F.E., Gonzalez, J. A., Boero, C.,
Gallardo, M., Boero, C. and Kortsarz, A., 1995, Changes in soluble carbohydrates and invertase activity in Chenopodium quinoa developed for saline stress during germination. Cur. Top. Phytochem., 14: 27-34.

Singh, P., Singh, N., Sharma, K. D. and Kuhad, M. S., 2010, Plant water relations and osmotic adjustment in Brassica species under salinity stress. $J$. Am. Sci., 6(6):1-4.

Younis, S. A., Shahatha, H. A., Hagop, P. G. and AI-Rawi, F. I., 1991, Effect of salinity on the viability of rice seeds. Plant Growth, Drought and Salinity in the Arab Region, pp: 235- 244.

Zapata, P. J. M., Serrano, M., Pretel, M. T., Amoros, A. and Botella, M. A. 2004, Plyamines and ethylene changes during germination of different plant species under salinity. Plant Sci., 167: 781-788.

www.ccari.res.in/wasp.

\section{How to cite this article:}

Rituparna Munshi, Ajanta Debbarma, Pinaki Acharyya, Arup Chattopadhyay, Pritam Mishra, Lopamudra Chakraborty and Biswanath Sahoo. 2019. Salt Induced Modulation on PhysioBiochemical Attributes in Okra (Abelmoschus esculentus (L.) Moench. Int.J.Curr.Microbiol.App.Sci. 8(06): 2883-2892. doi: https://doi.org/10.20546/ijcmas.2019.806.347 